\title{
Habitar, (ser-en) el mundo
}

\author{
Álvaro Luis López Limón \\ Universidad Autónoma de Zacatecas \\ limonyparra@gmail.com
}

\section{Advertencia}

La presente reflexión se inscribe en una investigación que convoca a cuatro grandes pensadores, Hannah Arendt, Emmanuel Levinas, Martin Heidegger y Peter Sloterdijk, alrededor de una interrogante que, por cierto, teníamos en el abandono, sobre el sentido de habitar el mundo. Hemos considerado que la casa se nos revelará como la «condición» de la condición humana — valga la redundancia- y el lugar hermenéutico para entendernos a nosotros mismos. En esta ocasión atenderemos únicamente aquellos aspectos derivados de las incipientes lecturas de Heidegger que irrumpen como interrogantes y actúan a modo de ideas-guía, ¿es posible mostrar en esta relación la estructura ser-en-el-mundo como el modo del ser del hombre? ¿Dasein, existencia y seren-el-mundo indican que el hombre está situado? ¿La cercanía que le otorga el sentido es el fundamento de tener cura, de cuidar de o procurarse de? 
Nuestra meditación no se agota en estas primeras cuartillas, hemos elegido -con sumo cuidado- nueve citas que intercalamos en tres fragmentos, que si bien es cierto no precisan -por el momento- una unidad exhaustiva, sí le asignan sentido a nuestra indagación. Cabe señalar que tal vez no le dirán nada al lector desinformado, que quizá espere encontrarse con un tríptico explicativo de los conceptos aquí tratados, mapa o itinerario de fácil comprensión. Algún riesgo se tendrá que enfrentar.

\section{Presentación}

Es conveniente precisar - por el momento- dos situaciones: la primera, que la existencia humana se desenvuelve en un espacio vivencial; de hecho, es el carácter espacial en el que el ser del hombre se manifiesta como hombre, en cuanto a la relación que se establece entre la existencia humana y su espacio. La segunda, que en la estructura social de nuestra vida cotidiana estamos familiarizados con las cosas, tratamos con éstas y es en la medida que tratamos con las familiares que las comprendemos. Esta comprensión de las cosas ocurre como compresión de vínculos de significación, por ello no encontramos las cosas como objetos aislados sino como algo significativo. De modo que, en este trato con las cosas, al mismo tiempo y de alguna manera, nos tenemos a nosotros mismos, ocurre ahí -sin percatarnos- una especie de tenerse a sí mismo. Tercero, son la familiaridad y el tenerse a sí mismo los dos rasgos de la compresión propuestos por Martin Heidegger y su bosquejo preliminar consiste en dejarse guiar por la primera orientación del ser en del 
ser-en-el-mundo (2003) ${ }^{1}$. La vivencia del entorno muestra en sí un determinado modo ser que está caracterizado por la familiaridad con el entorno y sus cosas entornadas. Este modo de ser inicialmente mostrado indica el modo en el que nos encontramos de entrada en el mundo y las cosas de este mundo. El ser-en significa pues una relación inicial con el mundo, que no es categorial sino existenciaria y que puede ser caracterizada como habitar el mundo familiar, como un estar entre el mundo. Así, el hombre mismo define su forma de ser como ser-en-el mundo.

A partir de este contexto y como preámbulo establecemos la primera afirmación y punto de partida de nuestro cometido. Habitar es la forma propia que le atañe al hombre en el mundo, que el mundo tiene una dimensión simbólica y espacial, al mismo tiempo que se define como lugar y horizonte de sentido. El habitar es esta cercanía, esta familiaridad, es la condición para que las cosas vuelvan a ser cosas, y el mundo vuelva a ser mundo; una especie de retorno a las cosas mismas.

Ahora es tiempo de que los dioses emerjan desde las cosas que habitamos... Rainer María Rilke

Ser hombre significa ser habitante del mundo en tanto que es el ahí del ser del hombre. Así, el ser tiene lugar a través del

1 Este bosquejo se encuentra en el parágrafo \$12, titulado «Bosquejo del estar-en-elmundo como estar-en como tal». 
hombre, es decir, el ser ocurre, acaece y cada vez que acontece tiene lugar, pues no hay acto sin lugar. Es así que en cada acto el hombre se descubre, pero es la apertura del espacio que esta acción abre en cuanto acontece, a esto se le llama espaciar. Veamos lo que nos dice Heidegger:

El espacio no está en el sujeto, ni el mundo está en el espacio. El espacio está, más bien, «en» el mundo, en la medida en que el estar-en-el-mundo, constitutivo del Dasein (ser-ahí), ha abierto el espacio. El espacio no se encuentra en el sujeto, ni el sujeto considera el mundo «como si» éste estuviera dentro de un espacio, sino que el «sujeto», ontológicamente bien entendido, es decir, el Dasein, es espacial en un sentido originario (2003: 81).

Estar-en es la expresión existencial del ser del Dasein y su constitución esencial es ser-en-el-mundo. Entendido así, el espacio es vivencial; un espacio abierto por la identificación y apropiación del ser que hace del mismo espaciar un existenciario fundamental. La noción de casa tiene -sin lugar a dudas- este carácter porque se enraíza existenciariamente en el ser mismo. Es el espacio de donde todas las cosas proceden y lo que hace a las cosas ser lo que son desde donde se proyecta el ser efectivo del hombre en la realidad física, cultural y social del mundo en el que nos desplegamos.

El habitar es esta cercanía de la que hablamos, esta familiaridad, y es la condición para que las cosas vuelvan a ser cosas y el mundo vuelva a ser mundo; un retorno a las cosas mismas.

El acto originario es un acto de mutua apropiación entre el ser y el hombre; acto de apropiación originario que 
Heidegger llama suceso, pero que también relaciona con «lo apropiado»o «lo propio». En este sentido, espaciar o «abrir espacio» se da a través de la apropiación del ser del hombre como ser-en-el-mundo, es decir, como un ser existente. Las cosas del mundo se acomodan al hombre en el espacio, definiéndose en este encuentro la forma de estar en la que el propio ser del hombre se encuentra. Podemos decir que todo encuentro con las cosas es encuentro de sí, porque cada encuentro como co-apropiación o apropiación originaria le permite al hombre saberse en su existencia. La forma de ser que tiene el hombre es encuentro en la apertura donde se descubre su existencia.

Asimismo, se da -al mismo tiempo-su especial forma de ser en tanto estar-en-el mundo como una habitación abierta por el ser en su apertura. El hecho de que esta apertura sea un encuentro como apropiación determina el carácter afectivo y, a su vez, da cuenta de la forma esencialmente afectiva del Dasein. «En la disposición afectiva, el Dasein ya está siempre puesto ante sí mismo, ya siempre se ha encontrado, no en la forma de una autopercepción, sino en la de un encontrarse afectivamente dispuesto» (Heidegger, 2003: 160). El lugar espaciado, la habitación, siempre será un lugar de encuentro y cercanía en la forma en la que se realiza el despliegue del hombre como él mismo, como una acción que funda espacio. Esto significa que en él la cercanía permite la manifestación y el decir mismo.

El ser del hombre en tanto estar-en-el-mundo es la situación que da al espaciar todo su sentido y es por la forma que se relaciona el ser con el mundo que podemos hablar del lugar como un encuentro, como un acto de co-apropiación. 
En virtud de este estar-en-el-mundo determinado por el «con», el mundo es desde siempre el que yo comparto con los otros. El mundo del Dasein es un mundo en común. El estar-en es un co-estar con los otros. El ser-en-sí intramundano de éstos [sic] es la coexistencia (Heidegger, 2003: 144).

El estar-en-el-mundo es diferente al de los demás entes porque el hombre se mueve en un mundo de significados y valores. Así como el hombre establece una dinámica entre él y el mundo que es dada por un hacer con sentido. Habitar es la forma propia que le atañe al hombre en el mundo; aquí, éste tiene una dimensión simbólica y espacial al mismo tiempo que se define como lugar y horizonte de sentido.

Habitar también significa poder hacernos un mundo a la manera humana que, a su vez, haciéndolo nos hace. Si esto es así, entonces ser y hacer se vinculan como hacer en tanto actuar, construir, fabricar y en cuanto al propio hacer al ser mismo del hombre, es decir, en su hacerse que le es propio, en su actuar como artesano, constructor y fabricante.

En este sentido, a través de la relación de cercanía y familiaridad entre el hombre y el mundo, es posible espaciar una habitación. Lo que dice el espacio habitado da cuenta del propio decir del hombre que lo crea y funda y que, al mismo tiempo, en el mismo hecho de esta creación, se funda a sí mismo apareciendo como un ser que es habitando. El espacio así mostrado será dotado de sentido estableciendo direcciones en nuestro camino y sobre todo definiendo nuestra propia forma de ser a través de estas orientaciones.

La relación que nos acerca a las cosas es aquella que las trata como puntos de referencia y como útiles para la vida cotidiana. Esta relación muestra la estructura ser-en-el-mun- 
do como el modo del ser del hombre. Así, para Heidegger, Dasein, existencia y ser-en-el-mundo son sinónimos; los tres términos indican el hecho de que el hombre está situado. Es la cercanía, en tanto familiaridad, la que otorga el sentido ésta es el fundamento de tener cura, de cuidar de o procurarse de, y es a partir de esta familiaridad que se entiende el espacio originario.

El hombre, en tanto ser espacial y en tanto existenciario, sólo puede darse desde el des-alejamiento y la dirección, que no es otra cosa que hacer desaparecer la lejanía de algo: acercarse en el sentido de hacer familiar. La cafetera que utilizo para servir el café es «a la mano» y es cercana porque me es familiar en tanto útil, pero esta cercanía no tiene nada que ver con la distancia en la que se encuentre respecto a mí; por ello, hablamos de que la cercanía, en tanto familiaridad de los útiles, es ajena a las medidas geométricas y es justo con respecto a esta cercanía como se muestra la espacialidad originaria. Cuando el Dasein se encuentra con lo «a la mano», determina de una cierta forma el espacio, y esta forma de determinar el espacio es en la familiaridad, la cual se explica a partir de la noción de des-alejamiento. Por ello, Heidegger hablará de que la esencia del Dasein consiste en ser desalejador:

Desalejar quiere decir hacer desaparecer la lejanía, es decir, el estar lejos de algo; significa, por consiguiente, acercamiento. El Dasein es esencialmente des-alejado; por ser el ente que es, hace que el ente comparezca viniendo a la cercanía. La desalejación descubre el estar lejos. El estar lejos, al igual que la distancia, es una determinación categorial del ente que no es el Dasein. En cambio, la desalejación debe ser entendida como un existencial (2003: 130). 
Sólo con respecto al Dasein algo es lejano, porque lo propio del Dasein, lo que le es esencial, es la cercanía y la presencia, no la magnitud de la distancia. El hombre o el ser-ahí (Dasein) tiene una tendencia esencial a la cercanía, pero esta cercanía nada tiene que ver con la medida de la distancia sino con la familiaridad y, por lo tanto, con la apropiación. La familiaridad no se podría entender sin la idea de cuidado, y esto porque la familiaridad cuida; por eso, el Dasein está en y es la forma en la que se desarrolla esta espacialidad.

En cambio, el Dasein está «en» el mundo en el sentido del ocupado y familiar habérselas con el ente que comparece dentro del mundo. $Y$, por consiguiente, si la espacialidad le corresponde en alguna forma, será únicamente sobre la base de este estar-en. Ahora bien, la espacialidad de este último presenta los caracteres de la des-alejación y la direccionalidad (Heidegger, 2003: 130).

Así pues, estar en el sentido del ocupado indica el modo peculiar en el que nos comportamos respecto al mundo en forma cotidiana. Sólo en el Dasein está implícita una esencial tendencia a la cercanía.

Únicamente el hombre puede dar mundanidad y, por lo tanto, significatividad o pertenencia a las cosas, ya que sólo de forma familiar éstas - mundanidad y significatividad- resultan propias del mundo y de él. Para Heidegger esta reflexión tomará el nombre de «la espacialidad de lo a la mano dentro del mundo» (2003: 127), como situación fundamental para comprender la relación del hombre con las cosas, lo que significa que la espacialidad se construye sobre la cercanía. 
Nuestro ser en el mundo no es simplemente estar inmersos en instrumentos, sino que se define por su familiaridad con un mundo de significados, por ser en éste donde se abre el mundo. El uso de las cosas está íntimamente relacionado con el significado que éstas tienen en nuestra vida; dicho significado no es algo que sólo se agregue al objeto material, sino que se integra como parte esencial de un todo concreto en el que comprometemos su uso en la existencia.

Lo específico de la cosa es actuar en la medida en que es parte de nuestro mundo de interactuación y en tanto que el instrumento es definido en su uso, en su significado y en su valor. De este modo, el uso se dará dentro del horizonte de significados al cual pertenece en el mundo de instrumentos. Un instrumento está hecho para servir a un fin dado y lo propio de éste consiste en constituirse en función de otros; es referencia de que no sólo incluye la utilidad que tiene, sino que habla de los materiales con los que está hecho y la región a la que pertenece, en otras palabras, se constituye como el sentido de la mundanidad. Esta referencia, siempre de algo que da el instrumento, es constitutiva y Heidegger la llamará como signos.

Pues bien, los signos son, en primer lugar, útiles, y su específico carácter pragmático consiste en señalar. Son signos en este sentido los hitos de los caminos, las piedras limítrofes en los campos, el globo anunciador del mal tiempo para la navegación, las señalizaciones, las banderas, los signos de duelo, etc. El señalar puede determinarse como una «especie» de remisión. Remitir, tomado en un sentido formalísimo, es relacionar (2003: 104). 
El signo siempre será un referirse a, es decir, una conexión con otra cosa. En tanto instrumentos, las cosas siempre nos están remitiendo a algo que no son ellas mismas, pero en el signo, este ser relación aparece en la identidad entre su uso y la capacidad de referencia, de manera que sirve como instrucción para el uso de los entes en su instrumentalidad. En este sentido, la relación y el conocimiento que tiene el hombre de y con las cosas como útiles no puede consistir en un ir del sujeto al objeto, ni en la interiorización del objeto en el sujeto, sino que es una interpretación. Pero no toda relación es un señalar, como lo dirá Heidegger, toda «remisión es una relación, pero no toda relación es una remisión. Toda 'señalización' es una remisión, pero no todo remitir es un señalar» (2003: 104).

Así, el referir que señala se funda en la forma de ser del útil como servir para, lo que determina su estructura óntica del qué. En contraparte, el servir para es la determinación ontológica del útil, donde el qué del ente aparece en el señalar. La casa, más que ser un útil que sirve para habitar, es una señal y un signo de que en él se habita mostrando su más propia manera de ser.

Observemos el ejemplo de Heidegger para explicar qué es un útil, esto es, unos zapatos de campesino que Van Gogh representa en unas de sus obras. En ellos se muestra su pertenencia al campo y a sus jornadas de trabajo, observándose así una manera de representar al mundo del cual forman parte.

Por este útil cruza el mudo temer por la seguridad del pan, la callada alegría de volver a salir de la miseria, el palpitar ante la llegada del hijo y el temblar ante la inminencia de la muerte en torno. Propiedad de la tierra es este útil y lo resguarda el mundo de la labriega. De 
esta resguardada propiedad emerge el útil mismo en su reposar en sí (Heidegger, 2003: 60).

Los zapatos son llevados a la tierra de cultivo, en donde realmente son lo que son; camina con ellos y así es como sirven los zapatos, esto es, los zapatos hacen de zapatos y no de objeto; están ahí acompañando en el campo y con ellos trabaja. Para Heidegger, lo más esencial del ser del útil es su ser de confianza, ya que por el intermedio de este útil se atiende la labor de la tierra

en virtud del ser de confianza del útil, está la labriega segura de su mundo. Mundo y tierra sólo existen para ella y para los que existen con ella de su mismo modo, sólo así: en el útil. [...] El ser útil, el ser de confianza, concentra en sí todas las cosas a su modo y según su alcance (2002: 61).

El útil sirve para algo, porque en él habita su ser de confianza y no al revés; si tomáramos en cuenta sólo su utilidad se perdería entonces su ser de confianza. La experiencia de la cercanía con las cosas, la que otorga la confianza en el instrumento, no es posible bajo el pensamiento técnico; éste no permite el acceso a esta experiencia, de ahí que tanto la cercanía como la lejanía sean improcedentes con la técnica. La supresión de la distancia no acerca, sólo lleva a una uniformidad en la que nada está ni cerca ni lejos.

La relación que tiene el hombre con las cosas es esencialmente apertura del hombre que permite que el ser se haga presente. El hombre no es una cosa entre cosas, sino que su esencia consiste en su apertura al ser. Lo que ve Heidegger frente a la técnica es la indigencia del pensar. De lo que se 
trata aquí es de ir más allá del pensamiento representador de la técnica. En esto consiste el Ge-stell para Heidegger; ésta es la estructura de emplazamiento de la técnica moderna, que saca de lo oculto lo real y efectivo como existencias (2001). En la experiencia del Ge-stell, también se encuentra la posibilidad de superar el dominio de esta estructura que convierte todo en existencia.

Finalmente, su propuesta es señalar otro tipo de relación entre el hombre y el ser, una relación de mutua apropiación. Para lograr establecer esta relación, el hombre debe reencontrar su esencia y habitar en ella; ésta esencia es la morada del ser, y la cercanía para con las cosas. Así, otorga la centralidad al ser cuando el útil no es solamente utensilio, sino que es en tanto que yo soy quien depende de él. Al experimentar la cosa, se deja la metafísica del sujeto, quien era el fundamento de toda la realidad; no es él quien constituye el mundo, sino la forma en que está vinculado a las cosas y al mundo al lado de ellas. Todo esto implica darnos cuenta de la cercanía que tenemos con las cosas y cuál es nuestra posición respecto de ellas.

\section{III}

Cuando Heidegger, citando a Hölderlin, afirma que el hombre habita poéticamente esta tierra, lo dice por la relación cercana hacia las cosas mismas; en otras palabras, el habitar poético se define por la cercanía. El hombre que habita de este modo deja a un lado la percepción de los objetos aislados, deja de ser un sujeto separado del objeto, porque se encuentra en lo más originario. Podemos decir que se encuentra en casa, cuya definición viene dada por la cercanía con las cosas. Sólo experimentando las cosas así dejamos atrás la metafísica del sujeto. No soy yo quien constituye el mundo 
de las cosas, sino que me encuentro vinculado a las cosas y, junto con ellas, al mundo. En este sentido, la centralidad está puesta en el ser y no en el Dasein: éste ya no determina al utensilio. Si pensamos en el ejemplo de los zapatos de Van Gogh, estos reúnen y muestran los elementos del mundo, las relaciones en las cuales el ser se muestra son, de hecho, la figura primordial del ser. Esta figura es llamada por Heidegger como la Cuaternidad o Cuaterna, relación entre tierra, cielo, mortales e inmortales. En este sentido, «la cosa se despliega como cosa. El desplegarse como cosa reúne. Reúne la Cuaterna: lo cuadrante» (1997: 234). Que la cosa llegue a ser cosa quiere decir que asciende ontológicamente, su cercanía hace que no sean meros objetos externos sino parte de la vida, llevando un ser más profundo en ellas.

Sólo la cercanía nos abre al mundo y evoca ser. Sólo en esta relación, el hombre está en lo más originario y fundamental que es la cercanía con las cosas. Pero la cercanía no es un ejercicio teorético, se lleva a cabo en la relación con las cosas, con el mundo y con los elementos que las constituyen. Pensar requiere experimentar, de modo que hay que situar tanto en el habitar humano como en la casa ese pensar que es, al mismo tiempo, experiencial. Hablar de casa es -yaapreciar la cercanía y encaminarse hacia ella.

El habitar es ser en el mundo junto con las cosas porque esencialmente estamos arrojados en el mundo. Su ser se desarrolla en el estar fuera, en otras palabras, el Dasein es un ser en el que su ser le va su ser mismo y lo propio de éste es estar lanzado.

Para Heidegger, construir es esencialmente habitar; así, construir no sólo es medio y camino para el habitar, sino que «el construir es en sí mismo ya el habitar» (2001a: 108), es la 
palabra misma la que muestra la esencia del habitar, de este modo, el hombre es en la medida en que habita.

El construir como el habitar, es decir, estar en la tierra, para la experiencia cotidiana del ser humano es desde siempre, como lo dice tan bellamente la lengua, lo 'habitual'. De ahí que se retire detrás de las múltiples maneras en las que se cumplimenta el habitar, detrás de las actividades del cuidar y edificar (Heidegger, 2001a: 109).

Heidegger pondrá mayor atención en este cuidar y en la cercanía de las cosas en las que es posible que las cosas sean cosas y que el mundo sea mundo. Sin embargo, esta forma de entender la construcción caerá en el olvido. En este giro es justamente donde la experiencia de habitar no se piensa como algo esencial del ser del hombre. Cuando construimos espacios lo hacemos habitándolos, viviéndolos.

El habitar humano puede tener dos direcciones, una en tanto la necesidad al interior del permanecer amparado, y otra, como un deseo en el hecho de morar, como un de-morarse en la protección. Pero este demorarse junto a la cercanía es una experiencia que no cabe en el mundo de la técnica por la falta de arraigo, de ahí que el pensar y el poetizar sean formas de un nuevo enraizamiento. Pero este pensar no es el que calcula y planifica, sino que lleva la experiencia de habitar las cosas y la cercanía, aquel que puede ver que el hombre no es una cosa entre cosas, sino que es apertura al ser, es decir, el que puede ver la co-pertenencia entre hombre y ser.

La experiencia -y relación fundamental- de la cercanía se encuentra en la Cuaterna, porque el genuino habitar acontece cuando los mortales protegen su esencia. 
Los mortales habitan en la medida en que salvan la tierra. [...] La salvación no sólo arranca algo de un peligro; salvar significa propiamente: franquearle a algo la entrada a su propia esencia. [...] Los mortales habitan en la medida en que reciben el cielo como cielo. Dejan al sol y a la luna seguir su viaje; a las estrellas su ruta; a las estaciones del año, su bendición y su injuria; no hacen de la noche día ni el día una carrera sin reposo. Los mortales habitan en la medida en que esperan a los divinos como divinos. Esperando les sostienen lo inesperado yendo al encuentro de ellos; esperan las señas de su advenimiento y no desconocen los signos de su ausencia. [...] Los mortales habitan en la medida en que conducen su esencia propia -ser capaces de la muerte como muerte- al uso de esta capacidad, para que sea una buena muerte. [...] En el salvar la tierra, en el recibir el cielo, en la espera de los divinos en el conducir de los mortales acaece de un modo propio el habitar como el cuádruple cuidar (mirar por) de la Cuaternidad (Heidegger, 2001a: 111).

Éste es el centro y la matriz de todo habitar, son los mortales cuya morada habita en la co-pertenencia a la Cuaternidad, es decir, al hombre le es propio el ser en las relaciones originarias que lo reúnen con la tierra, el cielo y lo divino. Lo anterior es posible por su esencial condición finita que se caracteriza por la apertura a lo otro de sí. Es el cuidado de preocuparse de lo esencial en el habitar humano. El cuidar por es velar por y en este sentido es cultivar, se trata de ofrecer residencia para poder permanecer en ella albergado, es decir, los modos de habitar la Tierra son los modos de construirla, de estos modos recibimos nuestra esencia que se conforma temporalmente. 
Así pues, la labor del hombre consiste en llamar a lo abierto, dejando reposar las cosas en lo que son; la creación es un llamado al recogimiento tanto en el reposo que alberga como en el cambio y el movimiento. «La cotidianidad media del $D a-$ sein puede ser definida, por consiguiente, como el estar-enel-mundo cadentemente abierto, arrojado-proyectante, al que en su estar en medio del 'mundo' y coestar con otros le va su poder-ser más propio» (Heidegger, 2003: 203-204).

Visto desde aquí, decir hombre es hacer referencia a una residencia que abre lugar a las cosas. «No hay los hombres y además espacio; porque cuando digo un hombre y pienso con esta palabra en aquel que es al modo humano, es decir, que habita, entonces con la palabra 'un hombre' estoy nombrando ya la residencia» (Heidegger, 2001a: 115-116).

El espacio da lugar y admite las cosas y la Cuaternidad, de manera que se instala en pertenencia el uno con el otro. Cuando esto sucede, el lugar es un cobijo o lo que es lo mismo, una casa. Las cosas del tipo de estos lugares dan casa a la residencia del hombre, de tal manera que la esencia del construir es, pues, dejar habitar.

[...] salvar la tierra, recibir el cielo, estar a la espera de los divinos, guiar a los mortales, este cuádruple cuidar es la esencia simple del habitar. De este modo, las auténticas construcciones marcan el habitar llevándolo a su esencia y dan casa a esta esencia (Heidegger, 2001a: 115-116).

El hombre mismo resulta un ser definido como lugar, así el Dasein es la casa del ser. En este sentido, la casa es fundamento, porque es el espacio originario en el que el ser se muestra. Por eso, habitar es ser-en el mundo. 


\section{A manera de conclusión}

Al caminar -de la mano de Heidegger-hemos logrado pensar el habitar humano y el hombre como ser-en-el-mundo, bajo una forma originaria de ser con las cosas y los hombres, es decir, bajo una relación de familiaridad y cercanía. Hoy sabemos que tanto estar en la casa como estar-en-el-mundo son los modos de ser que se fundan en la familiaridad y el cuidado, la preocupación o la cura hacia sí mismo y el mundo.

\section{Bibliografía}

Heidegger, M. (2003). Ser y Tiempo. España: Trotta.

Heidegger, M. (2002). El origen de la obra de arte. México: FCE. Heidegger, M. (2001b). Conferencias y artículos. España: Serbal.

Heidegger, M. (2001a). Conferencias y artículos. España: Serbal.

Heidegger, M. (1997). Filosofía, ciencia y técnica. Chile: Editorial Universitaria. 


\section{Resumen}

A través del pensamiento de Heidegger se recorre la interrogante del sentido de habitar en el mundo, partiendo del tenerse a sí mismo como sujeto y de la familiarización que tenemos los seres humanos con las cosas. Así, habitar es la forma que le concierne al ser humano en el mundo, es decir, la cercanía y la familiaridad con las cosas que dan retorno a las cosas mismas; se trata de una situación donde el ser humano se mueve en un mundo de significados y valores. Es la relación de utilidad de las cosas la que muestra la estructura del ser-en-el-mundo como manera de ser del hombre.

Heidegger señala otro tipo relación entre el ser humano y el ser, que es de mutua apropiación, donde el ser humano reencuentra su esencia y habita en ella, siendo la morada del ser. Además, es gracias a la cercanía que el ser humano tiene con las cosas que existe de manera poética.

Por tanto, decir hombre se refiere a una residencia que le abre lugar a las cosas, es un dejar habitar que, a su vez, dan casa a la residencia del hombre. En este sentido, la casa se convierte en fundamental, pues habitar es ser en el mundo, y la cura hacia sí mismo y el mundo.

Palabras clave: habitar, mundo, ser, familiar, relación, cosa, hombre.

\section{Abstract}

Through the thought of Heidegger, it is reviewed the question about the sense of inhabiting the world, starting with having yourself and the familiarization that human have with things. There for, inhabiting is the way that concerns the human being with the world, that is, the closeness and 
familiarity with things that they return to themselves; it is a situation in which human being moves around the world of meanings and values. The relationship of utility of the things shows the structure of being-in-world as a way of being of the human being.

Heidegger points out another type of relationship between human being and being, that is of mutual appropriation, here, the human being rediscovers his essence and live in it, being the abode of being. Also, thanks to closeness with things, the human being exists poetically.

So, to say men means a residence that opens a place to things, it is let inhabit which in turn give home to the residence of human being. In this sense, home is something fundamental, because inhabiting is being in the world, and it is the cure for yourself and the world.

Keywords: inhabiting, world, being, familiar, relationship, thing, human being. 
